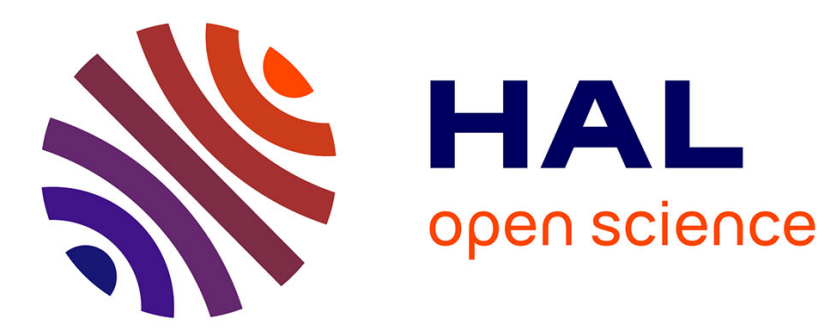

\title{
ULISS project: First comparison of cryocooled sapphire oscillators at the 10-15 level
}

S. Grop, B. Dubois, J. Masson, G. Haye, P.-Y. Bourgeois, Y. Kersalé, E. Rubiola, V. Giordano

\section{To cite this version:}

S. Grop, B. Dubois, J. Masson, G. Haye, P.-Y. Bourgeois, et al.. ULISS project: First comparison of cryocooled sapphire oscillators at the 10-15 level. IEEE International Frequency Control Symposium, May 2012, Baltimore, United States. pp.638-642, 10.1109/FCS.2012.6243705 . hal-00734596

\section{HAL Id: hal-00734596 \\ https://hal.science/hal-00734596}

Submitted on 4 May 2021

HAL is a multi-disciplinary open access archive for the deposit and dissemination of scientific research documents, whether they are published or not. The documents may come from teaching and research institutions in France or abroad, or from public or private research centers.
L'archive ouverte pluridisciplinaire HAL, est destinée au dépôt et à la diffusion de documents scientifiques de niveau recherche, publiés ou non, émanant des établissements d'enseignement et de recherche français ou étrangers, des laboratoires publics ou privés. 


\title{
ULISS project: First comparison of two Cryocooled Sapphire Oscillators at the $10^{-15}$ level
}

\author{
S. Grop ${ }^{\dagger}$, B. Dubois ${ }^{\dagger}$, J.-L. Masson*, , G. Haye* ${ }^{* \dagger}$ \\ , P.-Y. Bourgeois*, Y. Kersalé*, E. Rubiola*and V. Giordano*† \\ *FEMTO-ST Institute, Time and Frequency Dpt., 32 av. de L'Observatoire, 25044 Besançon Cedex, France \\ †ULISS-ST, SAIC Université de Franche-Comté, Témis Sciences, 25000 Besançon Cedex, France
}

\begin{abstract}
We already demonstrated a state-of-the-art Cryogenic Sapphire Oscillator (CSO), which incorporates a pulse-tube cooler instead of a bath cryostat - thus eliminating the need for regular supplies and manual transferring of liquid helium. The advent of reliable and cryocooled (CSO) open the possibility to implement such an ultra-stable reference not only in metrological laboratories with liquid helium facilities but also in remote sites like base stations for space navigation, VBLI antenna sites, ... The first prototype, i.e. ELISA, has been implemented in the ESA ground station in Malargüe (Ar) in April 2012. A second CSO was recently achieved using the same technology but specially designed to be transportable. After its complete assembly the CSO was coold down for the first time, and a preliminary frequency stability evaluation was done by direct comparison between the two CSOs. The Allan deviation was derived from data collected during more than 3 days. A short term frequency stability better than $2 \times 10^{-15}$ at 1 s was obtained. In spite of bad environemental conditions (infrastructure works in the building: implementation of an air conditioning in our laboratory which is not still effective) the frequency stability stays better than $4 \times 10^{-15}$ for $\tau<1,000 \mathrm{~s}$ and $1 \times 10^{-14}$ over one day.
\end{abstract}

\section{INTRODUCTION}

We recently developped a Cryogenic Sapphire Oscillator (CSO) named Elisa presenting a short term frequency stability better than $3 \times 10^{-15}$ for $1 \mathrm{~s} \leq \tau \leq 1000 \mathrm{~s}$ and achieving $4.5 \times 10^{-15}$ for one day integration [1], [2]. This CSO was designed and built in the framework of a research contract funded by the European Space Agency (ESA). It incorporates a pulse-tube cryocooler instead of a bath cryostat, thus eliminating the need for regular supplies and manual refilling of liquid helium. The advent of reliable and cryocooled CSO open the possibility to implement such an ultra-stable reference not only in metrological laboratories with liquid helium facilities but also in remote sites like base stations for space navigation, VBLI antenna sites, ... [3], [4]

In our project ULISS (Ultra Low Instability Signal Source), funded by Regional and European Institutions, we built a new cryocooled oscillator named ULISS specially designed to be transportable. The ULISS oscillator was already used to qualify with success a high stability frequency source located at Neuchâtel, Switzerland, and the PHARAO frequency synthesis Toulouse, France. ULISS was specially moved from FEMTOST for the measurement compaigns.

In this paper we present the frequency stability characterization of the newly built CSO demonstrating the reproductibility of our technology and we summarize the main results obtained at the LTF and CNES.

\section{SCHEMATIC ARCHITECTURE OF THE CRYOCOOLED SAPPHIRE OSCILLATOR INSTRUMENTS}

The CSO design and its characterization have been already described [1], [2], [5]-[7]. The heart of the system is a whispering gallery mode sapphire resonator made of a $54 \mathrm{~mm}$ diamter and $30 \mathrm{~mm}$ hight high purity sapphire cylinder placed in the center of a copper cavity. This assembly is thermally connected to the second stage of a pulse tube cryocooler specillay designed to ensure a high thermal stability $( \pm 1 \mathrm{mK})$ and a low level of mechanical vibration: axial displacement less than $2 \mu \mathrm{m}$ at the PT cycle frequency $(\approx 1 \mathrm{~Hz})$. The $\mathrm{CSO}$ is designed to oscillate at $9.99 \mathrm{GHz} \pm 5 \mathrm{MHz}$. The difference with the $10 \mathrm{GHz}$ round frequency is compensated by a low noise DDS. VHF and RF signals are synthetized from the stabilized $10 \mathrm{GHz}$. The system is complemented with a Phase-COmparator (PCO) enabling to lock the synthized outputs to an incoming $100 \mathrm{MHz}$ signal. The architecture of a Cryocooled Sapphire Oscillator Instrument is represented in the figure 1.

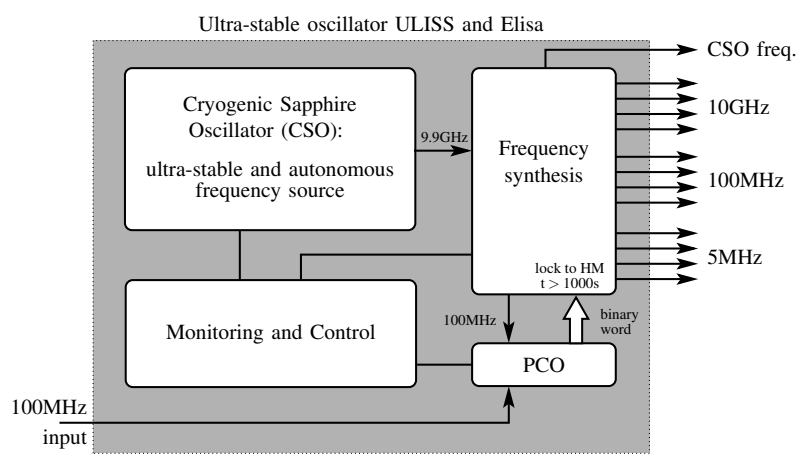

Fig. 1. Schematic architecture of a Cryocooled Sapphire Oscillator Instrument

\section{ULISS FREQUENCY STABILITY CHARACTERIZATION}

The ULISS unit similar to Elisa was finalized in November 2011. The two CSO outputs were mixed to generate a beatnote at $750 \mathrm{kHz}$. This beatnote was directly counted on an Agilent 53132A $\Lambda$-counter parametrized with a gate time $\tau=1 \mathrm{~s}$ [8], [9]. After approximately 4 days of acquisition, the relative 
frequency deviation $\sigma_{\Lambda}(\tau)$ was calculated for the different integration times $\tau$ by grouping the $1 \mathrm{~s}$ data. The result is given in the figure 2. No data post-processing has been done: neither abnormal point suppression nor drift removing.

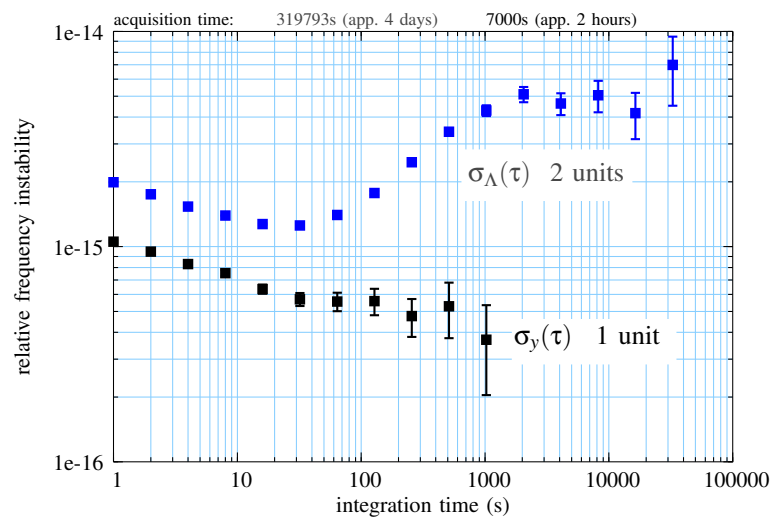

Fig. 2. Relative frequency stability of the $9.99 \mathrm{GHz} \mathrm{CSO}$ outputs

We measured a relative frequency stability $\sigma_{\Lambda}(\tau)$ better than $2 \times 10^{-15}$ for integration times $1 \mathrm{~s} \leq \tau \leq 200 \mathrm{~s}$. For longer integration times, we observed a hump around $2000 \mathrm{~s}$ and a drift of $1 \times 10^{-14} /$ day, which can be attributed to a residual CSO sensitivity to environmental perturbations. It should be mentionned that these measurements have been realized during infrastructure works in the building: implementation of an air conditioning system in our laboratory which is not still effective. This results is thus conservative. In spite of these bad metrological conditions, these performances demonstrate the reproductibility of our CSO technology.

The second curve of the figure 2 is the relative frequency deviation calculated from a quiet selected time period of about $7000 \mathrm{~s}$ extracted from the complete set of data. The calculated standard deviation corresponds to a flicker floor, i.e. its value does not depends on $\tau$. This flicker floor comes from the USO internal noise sources. As the two CSOs operate at a different frequency, we assume that these noise sources are decorrelated. In that case and if the two CSOs are assumed identical, it is justified to divide the result by $\sqrt{ } 2$ to obtain the frequency stability of one unit. Moreover the reference [9] gives the correspondance between $\sigma_{\Lambda}(\tau)$ and the true Allan deviation $\sigma_{y}(\tau)$. For white frequency of flicker frequency noise: $\sigma_{\Lambda}(\tau) \approx 1.3 \times \sigma_{y}(\tau)$. The open squares in the figure 2 represent $\sigma_{y}(\tau)$ evaluated taking into account these two corrections. The flicker floor of one unit is thus:

$$
\sigma_{y}(\tau)=4 \times 10^{-16} \text { for } 30 \mathrm{~s} \leq \tau \leq 500 \mathrm{~s}
$$

Although the following procedure has reasonable assumption and is often used to present USO characterization. It represents an optimistic evaluation. Remainder aware of this uncertainty, the flicker floor given in the equation 1 , can be considered as the best stability achievable by a well adjusted CSO in stable environmental conditions. The upper curve is the typical frequency stability achievable with our CSO in standard laboratory conditions.

\section{InStALlation OF ELISA IN DSA3}

Initially ELISA was build as a demonstrator to evaluate the possibility to get a high-frequency stability, i.e. better than $1 \times 10^{-14}$ at short term, without using liquid helium. As ELISA demonstrated very good performances surpassing the initial specifications, it was decided to implemented it in the new Deep Space Antenna Ground Station DSA3 in Malargue (Ar) still under construction. Thus all the instrument was conveyed by air to Buenos Aeres, then by truck to Malargue. About $30 \mathrm{~km}$ on an unpaved track enable to get eventually the ESA station.

The Frequency and Time system of the station consists in two active hydrogen masers and all the means to deliver the useful frequencies and to synchronize the antenna equipments. ELISA was integrated in this F\&T system as a complementary ultra-stable source for demonstration purpose. Only few days were necessary to restart ELISA and we conducted preliminary validation tests by comparing ELISA with the two HM. In this remote site we had not access at all the necessary instrumentation, and thus it was not possible to conduct a true 3-cornered-hat-method. Nevertheless we had to our disposal a commercial Phase-COmparator designed to compare Hydrogen Masers. The PCO does not allow to make a true cornered-hat evaluation as it enables only two synchronous phase diffrence measurements, i.e. $\varphi_{E}-\varphi_{H M 1}$ and $\varphi_{H M 2}-\varphi_{E}$. The third required estimation, i.e $\varphi_{H M 1}-\varphi_{H M 2}$, is obtained by summation of the two measured quantities. In such a case the instrumental noise is not rejected by the 3-cornered-hat process. The stability evaluation is thus limited at short term to $2 \times 10^{-14} / \tau$ due to the phase comparator intrinsic noise, which is not detrimental when qualifying Hydrogen Masers. In our case, the instrumental noise floor is clearly visible on the result (see Fig. 3).

This measurement has been done one week after the installation of the complete F\&T system. The two hydrogen masers and the CSO are running contunously since about one week, but have been greatly perturbed by the work in the experimental room (infrastructure work and cabling). Moreover the air-conditionning system was not at all tuned as the temperatures into the masers and CSO rooms are varying periodically over one hour of $2 \mathrm{Kpp}$. This situation should be greatly improved when all the instrumention will be installed.

\section{ULISS'S ODYSSEY}

\section{A. Test of an all-optical microwave signal generation}

The ULISS's Odyssey started the 15th February 2012 at the LTF of Neuchâtel. ULISS was operational three days later and was used as frequency reference to evaluate the frequency stability of a microwave signal generated from an optical frequency reference.

The optical frequency reference consists in a compact and low-cost planar waveguide external cavity laser (PW-ECL) 


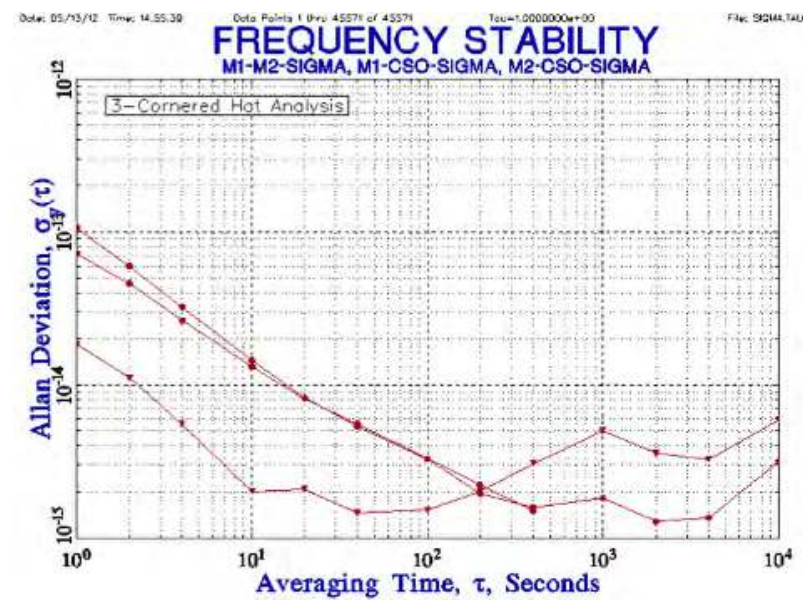

Fig. 3. Individual frequency stability of the three frequency standards of DSA 3: HM1, HM2 and ELISA. The dashen line indicates the noise floor of the PCO limiting the measurement to $2 \times 10^{-14} / \tau$.

stabilized on a high finesse Fabry-Perot ULE optical cavity. The frequency stability of this optical reference is transfered to microwave domain through optical-to-microwave frequency division with an femtosecond laser frequency comb. The comparison set-up is given in the figure 4 .

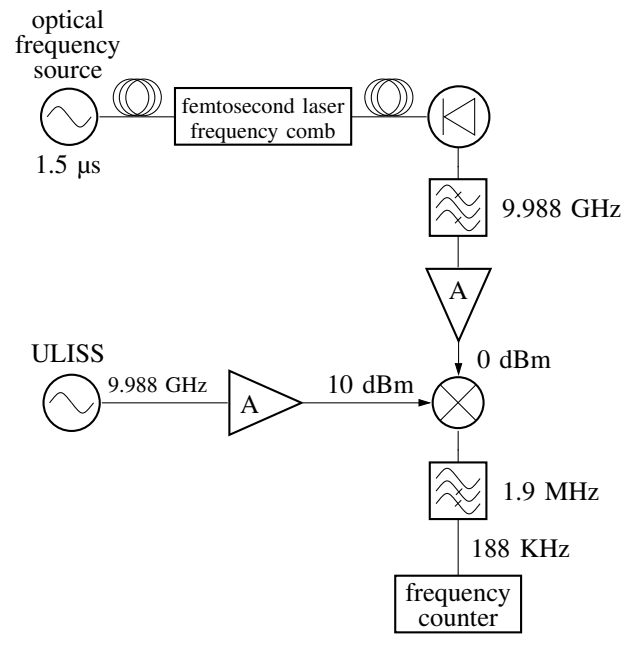

Fig. 4. Comparison set-up

The beam of the stabilized femtosecond laser was sent to a large bandwidth photodiode. The output signal of the photodiode is filtered to keep the 40th harmonic of the femtosecond laser repetition rate, amplified and mixed with the amplified $9.988 \mathrm{GHz}$ ultra-stable signal generated by ULISS. The resulting beatnote at $188 \mathrm{kHz}$ was counted to evaluate the frequency stability. The measurement result is shown in the figure 5.

ULISS allowed to evaluate the frequency stability of an all-optical microwave signal generator without the need of a

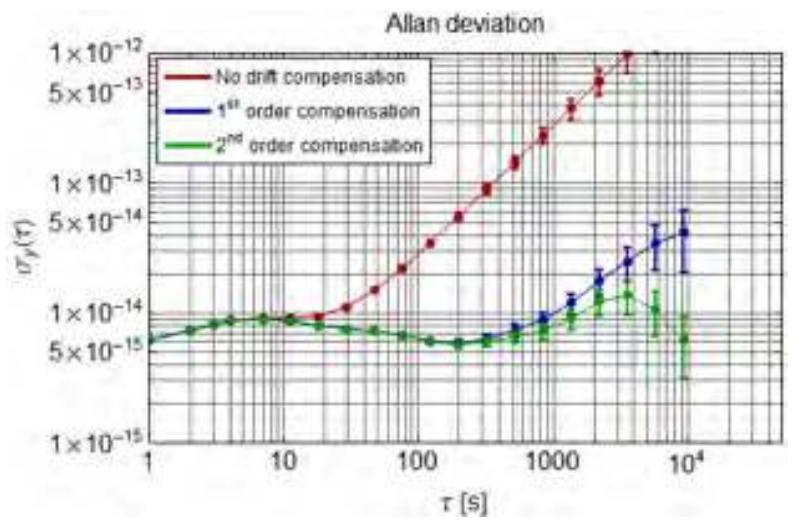

Fig. 5. Relative frequency stability of the $9.99 \mathrm{GHz}$ microwave signal generated with the femtosecond laser and ultra-stable laser

second equivalent unit.

\section{B. Test of an USO X-tal}

During the measurement compaign at the LTF, a quartz oscillator prototype from the Oscilloquartz company was characterized in term of frequency stability. The set-up measurement scheme is presented on figure 7 .

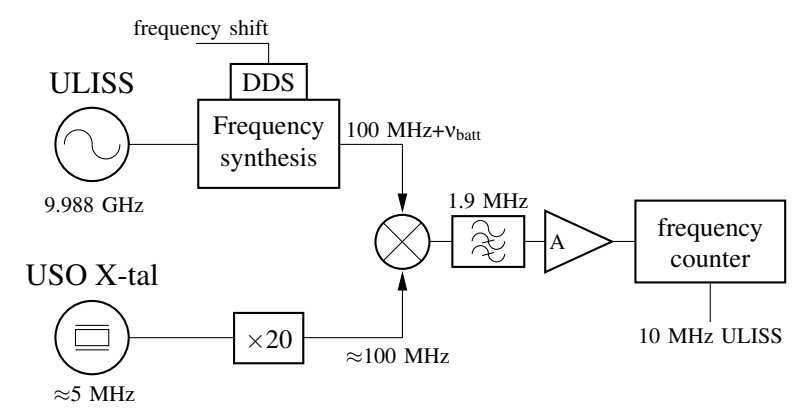

Fig. 6. Quartz oscillator frequency stability measurement set-up

To ensure a sufficient resolution of the measurement instrumentation, the quartz signal frequency was multiplied by 20 and compared to the $100 \mathrm{MHz}$ coming from the ULISS frequency synthesis. The best result is shown on figure 7 .

This is the second time that such a performance was observed unambiguously for a quartz crystal USO [10]. The frequency stability measured at the FEMTO-ST Institute in 2010 and at the LTF are identicals. It demonstrates the potentiality of ULISS to be used to qualify high-performances industrial products.

The 5th May, ULISS returned back to the FEMTO-ST Institute and was tested against Elisa to checked if any malfunctions appeared due to the trip. The 9th May, the same frequency stability as our first measurement compain was measured (figure 8).

This result is enough to prove the robustness and reliability of our ultra-stable frequency source. 


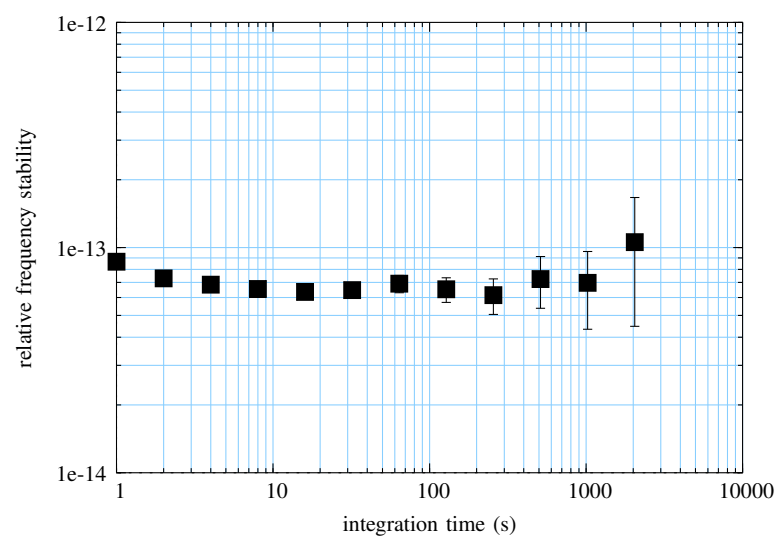

Fig. 7. Relative frequency stability of the best quartz oscillator

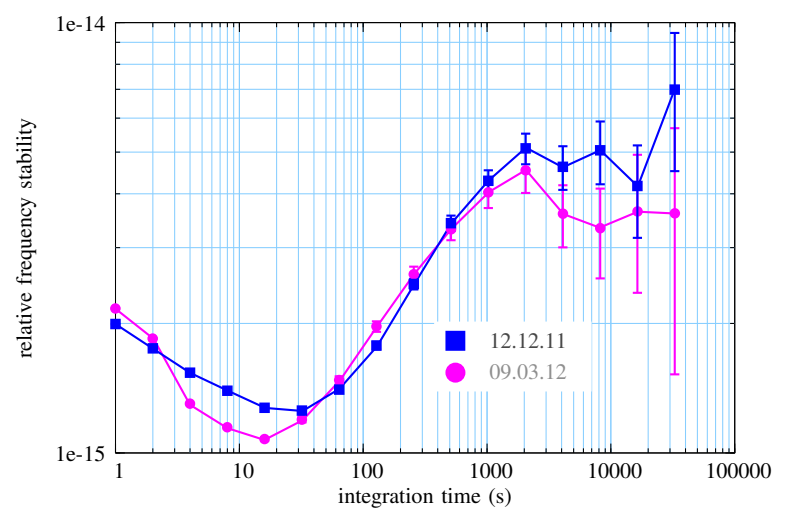

Fig. 8. Relative frequency stability of the $9.99 \mathrm{GHz} \mathrm{CSO}$ output before and after the trip to the LTF at Neuchâtel

\section{Test of an ultra-low noise microwave frequency synthesis}

The PHARAO project aimed to operate a cold-atoms caesium clock in microgravity in the International Space Station (ISS). The performances of this cold-atoms clock will be combined with those of an active Hydrogen Maser (HM) to generate an onboard timescale using the excellent short-term stability of the HM and the long-term stability and accuracy of the cesium clock PHARAO. This assembly constitutes the core of the ACES (Atomic Clock Ensemble in Space) instrument. Today, the PHARAO instrument is completing its qualification. ULISS was thus be used to qualify the flying model of the PHARAO 9.192GHz local oscillator. The signal that will probe the cold atoms is generated from a frequency synthesis referenced on a state-of-the-art quartz oscillator. Apart from the 9.192GHz signal, the PHARAO's frequency synthesis delivers a high frequency stable $100 \mathrm{MHz}$ signal to compare PHARAO to the HM. Drastic phase noise specification has been imposed on these two outputs. The use of ULISS as a frequency reference greatly simplfied the validation of the PHARAO's frequency synthesis. The figure 9 represents the phase noise measurement set-up used to characterize the $9.192 \mathrm{GHz}$ output signal.

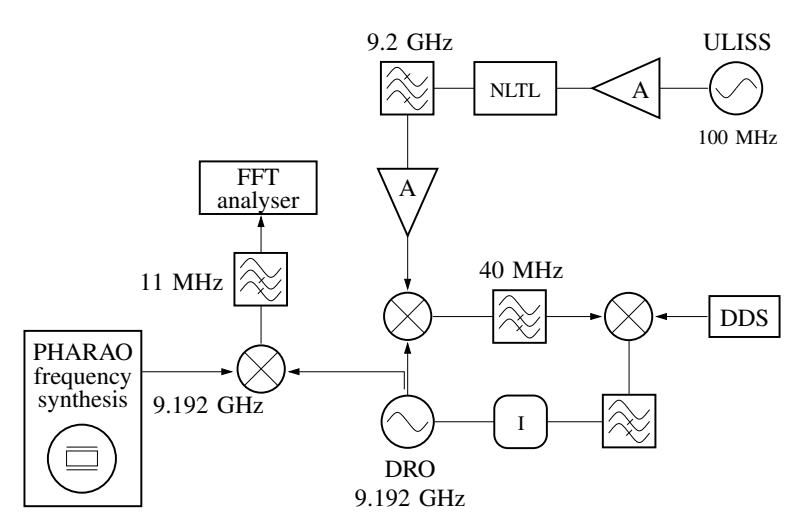

Fig. 9. Set-up of the $9.192 \mathrm{GHz}$ signal generation and phase noise measurement

A nonlinear transmission line (NLTL) generates the harmonics of the incoming $100 \mathrm{MHz}$. The $9.2 \mathrm{GHz}$ harmonic is filtered and compared to the signal of a $9.192 \mathrm{GHz}$ Dielectric Resonator Oscillator (DRO). A Direct Digital Synthesizer compensates for the frequency difference and is used to phase lock the DRO with a bandwidth of $300 \mathrm{kHz}$. The comparison with the PHARAO $9.192 \mathrm{GHz}$ is shown in the figure 10

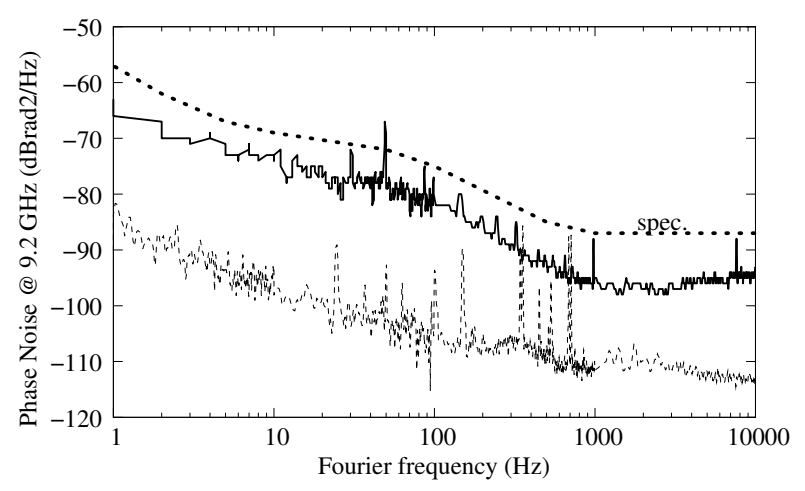

Fig. 10. Phase noise of the PHARAO frequency synthesis at $9.192 \mathrm{GHz}$ (bold line).

The $100 \mathrm{MHz}$ phase noise measurement set-up is far simpler. The $100 \mathrm{MHz}$ output of ULISS is adjusted, by shifting the frequency output of the ULISS's frequency synthesis DDS, to perfectly fit to the $100 \mathrm{MHz}$ output of the PHARAO's frequency synthesis, with the use of a commerciall phase comparator (Timetech). The result is shown in the figure 11 .

The measured phase noise is in accordance with teh expectations; Therefore, ULISS will be requested by CNES for the next steps of the PHARAO qualification.

\section{CONCLUSION}

The results shown on this paper demonstrate the technology maturity and reliability of our CSOs. We reached our objective to develop a transportable version of the stateof-the-art crycooled sapphire oscillator Elisa with equivalent 


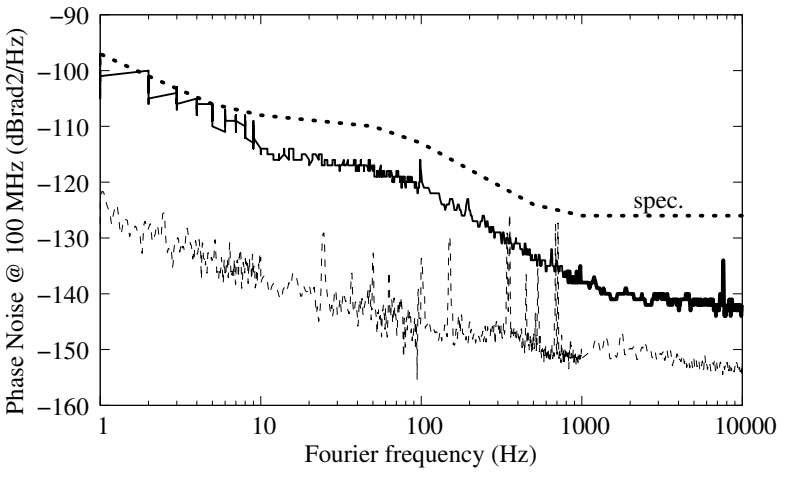

Fig. 11. Phase noise of the PHARAO frequency synthesis at $100 \mathrm{MHz}$ (bold line).

performances and we already visited two scientific institutes which led to interesting collaboration and results.

\section{ACKNOWLEDGMENT}

The authors wish to acknowledge to the Fond Européen de Développement Régional (FEDER), le Conseil Général de Franche-Comté and OSEO for their support, Jacques CHAUVIN from OSA to provide USO X-tal during the test compain at the LTF, Gianni DI DOMENICO, Stéphane SCHILT, Vladimir DOLGOVSKIY and Nikola BUCALOVIC from the LTF to provide their results and Claude ESCANDE, David VALAT and Sébastien TELLIER from CNES to provide the phase noise curves of the engineering model of the PHARAO frequency synthesizer.

\section{REFERENCES}

[1] S. Grop, P.-Y. Bourgeois, N. Bazin, Y. Kersalé, E. Rubiola, C. Langham, M. Oxborrow, D. Clapton, S. Walker, J. DeVicente and V. Giordano, Elisa: a cryocooled sapphire oscillator with $1.10-15$ frequency stability, Review of Scientific Instruments, 81, 2010

[2] S. Grop, W. Schafer, P.-Y. Bourgeois, Y. Kersalé, M. Oxborrow, E. Rubiola and V. Giordano, Unprecedented High Long Term Frequency Stability with a Microwave Resonator Oscillator, IEEE Transactions on Ultrasonics, Ferroelectrics and Frequency Control, 58, 8, 1694-1697, 2011.

[3] V. Giordano, S. Grop, P.-Y. Bourgeois, Y. Kersalé, E. Rubiola, M Mrad, C. Langham, M. Oxborrow and W. Schafer, Cryogenic sapphire microwave oscillators for space, metrology and scientific applications, General Assembly and Scientific Symposium, URSI, Istanbul, Turkey, 2011.

[4] J. G. Hartnett, N. R. Nand, Ultra-low vibration pulse-tube cryocooler stabilized cryogenic sapphire oscillator with 10-16 fractional frequency stability, IEEE Trans. on MTT, 58, 3580-3586, 2010.

[5] S. Grop, P.-Y. Bourgeois, R. Boudot, Y. Kersalé, E. Rubiola and V. Giordano, $10 \mathrm{GHz}$ cryocooled sapphire oscillator with extremely low phase noise, Electronics Letters, 46, 6, 420-422, 2010.

[6] S. Grop, W. Schafer, P.Y. Bourgeois, Y Kersalé, M Oxborrow, E. Rubiola and V. Giordano, Unprecedented High Long Term Frequency Stability with a Microwave Resonator Oscillator, IEEE Transactions on Ultrasonics, Ferroelectrics and Frequency Control, 58, 8, 1694-1697, 2011.

[7] S. Grop, P.-Y. Bourgeois, E. Rubiola, W. Schafer, J. DeVicente, Y. Kersalé and V. Giordano, Frequency Synthesis Chain For The ESA Deep Space Network, Electronics Letters, 47, 6, 386-388, 2011.

[8] E. Rubiola, On the measurement of frequency and of its sample variance with high-resolution counters, Review of Scientific Instruments, 76, 5, 2005 .
[9] S. T. Dawkins, J. J. McFerran and A. N. Luiten, Considerations on the measurement of the stability of oscillators with frequency counters, IEEE Transactions on Ultrasonics, Ferroelectrics and Frequency Control, 54, 5, 918-925, 2007.

[10] S. Grop, B. Dubois, P.-Y. Bourgeois, Y. Kersalé, E. Rubiola, G. Haye and V. Giordano, ULISS: A Mobile Cryogenic U ltra- Stable Oscillator, Joint conference of the 2011 IEEE IFCS-EFTF, San Francisco, US, 2011 